\title{
Long noncoding RNA expression analysis reveals the regulatory effects of nitinol-based nanotubular coatings on human coronary artery endothelial cells
}

This article was published in the following Dove Press journal:

International Journal of Nanomedicine

\author{
Pan Shang ${ }^{l} *$ \\ Gan Chen ${ }^{\prime} * *$ \\ Guannan $\mathrm{Zu}^{2}$ \\ Xiang Song' \\ Peng Jiao ${ }^{2}$ \\ Guoxing You' \\ Jingxiang Zhao' \\ Hongyi $\mathrm{Li}^{2}$ \\ Hong Zhou' \\ 'Academy of Military Medical Sciences, \\ Institute of Health Service and \\ Transfusion Medicine, Beijing 100850 , \\ People's Republic of China; \\ 2Photoelectrochemical Research Group, \\ School of Materials Science and \\ Engineering, Beijing University of \\ Technology, Beijing 100I24, People's \\ Republic of China \\ *These authors contributed equally to \\ this work
}

Correspondence: Hongyi Li

Photoelectrochemical Research Group,

School of Materials Science and

Engineering, Beijing University of

Technology, Pingleyuan 100\#, Chaoyang

District, Beijing 100124, People's Republic

of China

Email Ihy06@bjut.edu.cn

Hong Zhou

Institute of Health Service and

Transfusion Medicine, Academy of Military

Medical Sciences, Taiping Road 27\#,

Haidian District, Beijing, 100850, People's

Republic of China

Tel +8666930962

Fax +8666931970

Email zhouhtt1966@163.com
Background: Cardiovascular disease (CVD) is the leading cause of mortality all over the world. Vascular stents are used to ameliorate vascular stenosis and recover vascular function. The application of nanotubular coatings has been confirmed to promote endothelial cell (EC) proliferation and function. However, the regulatory mechanisms involved in cellular responses to the nanotubular topography have not been defined. In the present study, a microarray analysis was performed to explore the expression patterns of long noncoding RNAs (lncRNAs) in human coronary artery endothelial cells (HCAECs) that were differentially expressed in response to nitinol-based nanotubular coatings.

Materials and methods: First, anodization was performed to synthesize nitinol-based nanotubular coatings. Then, HCAECs were cultured on the samples for $24 \mathrm{~h}$ to evaluate cell cytoskeleton organization. Next, total RNA was extracted and synthesized into cRNA, which was hybridized onto the microarray. GO analysis and KEGG pathway analysis were performed to investigate the roles of differentially expressed messenger RNAs (mRNAs). Quantitative realtime reverse-transcription polymerase chain reaction (qRT-PCR) was performed to validate the expression of randomly selected lncRNAs. Coexpression networks were created to identify the interactions among lncRNAs and the protein-coding genes involved in nanotubular topographyinduced biological and molecular pathways. Independent Student's $t$-test was applied for comparisons between two groups with statistical significance set at $p<0.05$.

Results: 1085 lncRNAs and 227 mRNAs were significantly differentially expressed in the nitinolbased nanotubular coating group. Bioinformatics analysis revealed that extracellular matrix receptor interactions and cell adhesion molecules play critical roles in the sensing of nitinol-based nanotubular coatings by HCAECs. The TATA-binding protein (TBP) and TBP-associated transfactor 1 (TAF1) are important molecules in EC responses to substrate topography.

Conclusion: This study suggests that nanotubular substrate topography regulates ECs by differentially expressed lncRNAs involved extracellular matrix receptor interactions and cell adhesion molecules.

Keywords: nitinol titanium dioxide nanotubes, RNA sequencing, long noncoding RNA, nanotopography, molecular networks

\section{Introduction}

Cardiovascular disease is the leading cause of death all over the world. ${ }^{1}$ Various cardiovascular prostheses are often employed to treat and reconstruct damaged cardiovascular structures, such as cardiovascular vessels and heart tissues. 
However, clinical complications of the prostheses, such as in-stent restenosis and late stent thrombosis, frequently occur after implantation. Although numerous surface modification strategies have been developed, preventing these complications remains challenging.

Previous studies have demonstrated that rapid endothelialization on the surfaces of vascular prostheses is the most effective way to inhibit the above complications. ${ }^{2}$ There are many factors regulating rapid endothelialization on the surface of a prosthesis. ${ }^{3}$ One of the most significant factors is the interaction between the biomaterial surface and the ECs. It has been reported that the rate and quality of endothelialization on vascular prostheses depend on these interactions. ${ }^{4}$ Many factors regarding cell-substrate interactions are known to affect endothelial cellular adhesion, proliferation, spreading and other processes. Surface topography is a critical parameter among these factors. ${ }^{5}$ Numerous vascular stents with various topographic features have been designed to study the interactions between ECs and the cell-substrate interface. ${ }^{6}$ One of the most promising designs involves an upright nanotubular coating on the surface of a metallic substrate created via a simple anodization process. ${ }^{7}$ Several studies have investigated the behaviors and functions of ECs in response to nanotubular topography with titanium nanotubes. ${ }^{7-9}$ Nitinol is the universal clinical vascular stent material because of its superelasticity and blood compatibility; however, there have been few studies on nitinol nanotubes, although one recent study has shown that nitinol-based nanotubular coatings can improve endothelialization by promoting cell migration and the spreading of human aortic endothelial cells. ${ }^{10}$ Nevertheless, the mechanisms regulating the expression of genes in ECs responding to nitinol-based nanotubular coatings have not been defined.

Recent studies have shown that lncRNAs are functional RNA molecules longer than 200 nucleotides that are generally not translated into proteins but can regulate the expression and function of protein-coding genes by different mechanisms. ${ }^{11}$ Many lncRNAs have been implicated in cardiovascular pathophysiology and have been inferred as potential therapeutic targets. ${ }^{12}$ Recent evidence indicates that IncRNAs modulate the proliferation and migration phenotype of ECs, and aberrant expression of IncRNAs is closely associated with EC function $^{13}$ and death. ${ }^{14}$ Therefore, it is significant to determine the roles of IncRNAs in promoting EC proliferation and function in response to nitinol-based nanotubular coatings.
In previous studies, we investigated the effect of $\mathrm{TiO}_{2}$ nanotubes on the osteogenic differentiation process and the biological attachment mechanism of bone implants. ${ }^{15,16}$ On this basis, we are interested in the roles of lncRNAs in $\mathrm{EC}$ responses to nanotubular topography. Therefore, we cultured HCAECs on nitinol-based nanotubular coatings and utilized RNA-sequencing technology to analyze the expression patterns of lncRNAs and mRNAs in the HCAECs. Microarrays revealed that the nitinol nanotubes enhanced HCAEC motility via the sensing of nanotopographical cues by molecules involved in extracellular matrix receptor interaction and cell adhesion. Networks generated from significantly differentially expressed lncRNAs indicated that TBP and TAF1 are critical molecules in the interaction of ECs with nitinol-based nanotubular topography. Our results confirmed that 1085 differentially expressed lncRNAs participate in the regulation of HCAECs induced by nitinol-based nanotubular coatings.

\section{Material and methods}

\section{Synthesis and characterization of} nitinol-based nanotubular coatings

Nitinol foils were purchased (58.72 wt $\% \mathrm{Ni}$, superelastic, pickled surface, PEIER, Jiangyin, China) and were cut into $2 \times 2-\mathrm{cm}$ pieces. Then the nitinol foils were cleaned by ultrasonication in acetone and ethanol (Sinopharm Group Co., Ltd, Beijing, China). The foils were dried under nitrogen, and the control nitinol substrates and nanotubular substrates were labeled $\mathrm{CK}$ and NT, respectively. Anodization was conducted at $20^{\circ} \mathrm{C}$ in a Teflon container. The nitinol foil is the working anode while graphite foil is the counter electrode. Anodization was performed as described in our previous study on titanium nanotubes, ${ }^{17}$ with slight modifications. Briefly, the electrolyte contained $2 \mathrm{~g}$ of $\mathrm{NH}_{4} \mathrm{~F}$ (Sinopharm Group Co., Ltd), $1000 \mathrm{~mL}$ of ethylene glycol (Sinopharm Group Co., Ltd) and $10 \mathrm{~mL}$ of Millipore water. The anodization voltage was $30 \mathrm{~V}$ and the duration was $30 \mathrm{~min}$. Nanotubular substrates were rinsed in ethanol and Millipore water after anodization. Samples were subsequently annealed in a muffle roaster (CINITE, Beijing, China) for $2 \mathrm{~h}$ at a temperature of $500{ }^{\circ} \mathrm{C}$ to crystallize the nanotubes. Each substrate was sterilized with $75 \%$ ethanol and UV radiation before cell culture. The nanotubular coatings were observed with a scanning electron microscope (JEOL-JSM7001F, Tokyo, Japan). In addition, surface hydrophilicity was evaluated by 
measuring water contact angles (DataPhysics OCA15Pro, Filderstadt, Germany). Because nanotubular arrays are directly grown on nitnol sheets with $0.3 \mathrm{~mm}$ thickness and opaque, which is far more than the $100 \mathrm{~nm}$ thickness required for transmission electron microscope (TEM) study. Therefore, the nanotubular arrays on nitinol sheets can not support TEM study. The diagram is shown in $\underline{\text { Figure S1. }}$

\section{Cellular experiments}

HCAECs were purchased (ScienCell, San Diego, California, USA) and cultured under the manufacturer's instructions. Cells in passages 2-5 were used. The cell culture medium for the HCAECs was Endothelial Cell Medium (ScienCell) supplemented with endothelial cell growth factor, 20\% FBS and $100 \mathrm{U} / \mathrm{mL}$ penicillin/ $100 \mu \mathrm{g} / \mathrm{mL}$ streptomycin. Cells were cultured in an incubator containing $5 \%(\mathrm{v} / \mathrm{v}) \mathrm{CO}_{2}$ at $37{ }^{\circ} \mathrm{C}$ and culture medium was renewed every 2 days. For the extraction of total RNA, HCAECs were seeded onto the substrates, without protein pre-coating, and were then cultured for $48 \mathrm{~h}$.

To evaluate cell cytoskeleton organization, HCAECs were cultured on the samples for $24 \mathrm{~h}$ based on previous article $^{7}$ and were then fixed with 4\% paraformaldehyde (Sigma-Aldrich, St. Louis, MO, USA) in PBS for $15 \mathrm{~min}$. Then the cells were permeabilized using $0.1 \%$ Triton X-100 (Sigma-Aldrich) in PBS containing 1\% bovine serum albumin (Sigma-Aldrich) for $10 \mathrm{~min}$. Subsequently, the cells were labeled for F-actin using phalloidin conjugated to tetramethyl rhodamine isothiocyanate (Sigma-Aldrich) for $20 \mathrm{~min}$. Also, the cell nuclei were stained with 4-6-diamidino-2-phenylindole (Invitrogen, Carlsbad, USA) for $15 \mathrm{~min}$. After staining, the samples were washed three times in PBS. Finally, the samples were mounted on microscope slides for observation with a confocal laser scanning microscopy (Nikon A1, Tokyo, Japan).

To confirm the difference in cell phenotype between the CK and NT groups, immunofluorescent analyses of CD31, vWF, and vinculin were conducted. HCAECs were cultured on the samples for $72 \mathrm{~h}$, and then fixed with 4\% paraformaldehyde (Sigma-Aldrich) in PBS for 15 min. Then, the cells were permeabilized using $0.1 \%$ Triton X-100 (Sigma-Aldrich) in PBS containing 1\% bovine serum albumin (Sigma-Aldrich) for $10 \mathrm{~min}$. Next, the cells were incubated with CD31 mAb (Cell Signaling Technology, Danvers, MA, USA), vWF polyclonal antibody (Proteintech, Chicago, USA), and vinculin $\mathrm{mAb}$
(Proteintech) at $4{ }^{\circ} \mathrm{C}$ overnight. Subsequently, the cells were stained with Alexa Fluor 488/555-conjugated secondary antibody (Cell Signaling Technology). The cell nuclei were also stained with 4',6-diamidino-2-phenylindole (Invitrogen) for $15 \mathrm{~min}$. Following each step, the cells were washed three times with PBS. After that, the expression of adhesion molecules was determined by confocal laser scanning microscopy (Nikon A1).

For quantification of cytoskeletal features, ImageJ software was used to quantify cytoskeletal elongation. Elongation was quantified by measuring the long and short axes of the cell and calculating the aspect ratio (long to short axis ratio). Ten cells in each group were randomly selected for statistical analysis.

\section{Microarray analysis}

Total RNA was extracted from each sample in TRIzol Reagent (TaKaRa, Tokyo, Japan) with an Ultrapure RNA Kit (CWBIO, Beijing, China) under the manufacturer's instructions. Total RNA was quantified with a NanoDrop ND-2000 (Thermo Scientific, Waltham, MA, USA). Briefly, total RNA was synthesized into double-stranded cDNA. Then the cDNA was synthesized into cRNA labeled with Cyanine3-CTP, which was hybridized onto the microarray. After washing, the arrays were scanned with an Agilent G2505C Microarray Scanner (Agilent Technologies, Palo Alto, USA).

The array images were analyzed with Feature Extraction software (version 10.7.1.1, Agilent Technologies). The raw data were normalized via quantile algorithm and analyzed with the Agilent GeneSpring (version 14.8, Agilent Technologies). The probes that had at least 1 condition out of 2 conditions flagged as " $p$ " were chosen for further analysis. Then differentially expressed genes and lncRNAs were identified by fold changes and $p$-values calculated with t-tests. The threshold for upregulated and downregulated genes was a fold change $\geq 2.0$ and a $p$-value $\leq 0.05$. Finally, hierarchical clustering was conducted to display the expression patterns of the distinguishable genes among the samples.

\section{Quantitative real-time PCR validation}

qRT-PCR was carried out with a Bio-Rad thermocycler and SYBR Green Real-Time PCR Master Mix (Toyobo, Tokyo, Japan) under the manufacturers' instructions. The reaction conditions were as follows: incubation at $95{ }^{\circ} \mathrm{C}$ for $1 \mathrm{~min}$, followed by 40 cycles of $95{ }^{\circ} \mathrm{C}$ for $10 \mathrm{~s}, 60{ }^{\circ} \mathrm{C}$ for $15 \mathrm{~s}$ and $72{ }^{\circ} \mathrm{C}$ for $20 \mathrm{~s}$. The relative lncRNA and mRNA expression levels were calculated using the $2^{-\Delta \Delta \mathrm{Ct}}$ method. The 
expression levels of all targets were normalized to glyceraldehyde-3-phosphate dehydrogenase (GAPDH). The primer information could be found in Table S1.

\section{GO analysis and KEGG pathway analysis}

GO analysis and KEGG pathway analysis were performed to investigate the roles of differentially expressed mRNAs. Briefly, GO analysis was conducted to illustrate the genetic regulatory networks in accordance with three integrated domains (molecular function, biological process, and cellular component). The number of differentially expressed genes included in each GO term was counted, and Hypergeometric cumulative distribution function was used to calculate the enrichment of functional terms. The smaller the $p$-value, the more enriched the differentially expressed gene was in the GO term. The top 20 terms were sorted according to the $p$-value. Pathway analysis of differentially expressed genes was performed using the KEGG database, and Hypergeometric cumulative distribution function was used to calculate the enrichment of pathway terms. Again, the top 20 terms were sorted according to the $p$-value.

\section{Construction of the coexpression network}

Coexpression networks were created to identify the interactions among lncRNAs and the protein-coding genes involved in nanotubular topography-induced biological and molecular pathways. On the basis of Guttman et $\mathrm{al}^{18-20}$, IncRNAs that participate in special biological pathways are regulated by critical transcription factors (TFs) of those pathways. In order to determine whether the IncRNAs could possess transregulating functions, we compared the mRNAs that are coexpressed with lncRNAs with particular TFs regulated mRNAs. To investigate the mutual regulatory relationships among miRNA, IncRNA, and mRNA, we performed miRNAIncRNA-mRNA coexpression network analysis based on Pearson's correlation coefficient. Coexpression relationships with more than three shared miRNAs at $p<0.05$ were selected to build the miRNA-lncRNA-mRNA interaction network. The networks were constructed using the hypergeometric cumulative distribution function in MATLAB 2012b (The MathWorks, Natick, USA). The graphs were generated in Cytoscape 3.01 (Agilent Technologies).

\section{Statistical analysis}

All data were shown as the mean \pm standard deviation and were performed using SPSS 23.0 (SPSS Inc., Chicago, USA) statistics software. Student's $t$-test was applied to comparisons between two groups. A $p$-value $<0.05$ was considered statistically significant.

\section{Results}

\section{Material characterization}

The morphology of the nitinol-based nanotubular coatings was characterized by scanning electron microscope and is shown in Figure 1A and B. The typical outer diameter of the hollow nanotubes fabricated in this study was $\sim 50 \mathrm{~nm}$. The wettability of the implant surface plays a significant role in cell adhesion, ${ }^{21}$ so the surface hydrophilicity was evaluated by the measurement of the water contact angle. As shown in Figure $1 \mathrm{C}$, the contact angle of the water on the planar nitinol surface without anodic oxidation was $77.8 \pm 0.7^{\circ}$. After anodic oxidation and thermal treatment, the contact angle of the water decreased to $12.7 \pm 0.2^{\circ}$, indicating that the nitinol-based nanotubular surface was more hydrophilic than the planar nitinol surface.

\section{Cytoskeletal organization and phenotypic expression}

In general, cells undergo an adhesion process that involves substrate attachment, spreading, and cytoskeletal development. ${ }^{22}$ We investigated the cytoskeletal organization of HCAECs grown on planar nitinol (CK) and nanotubular

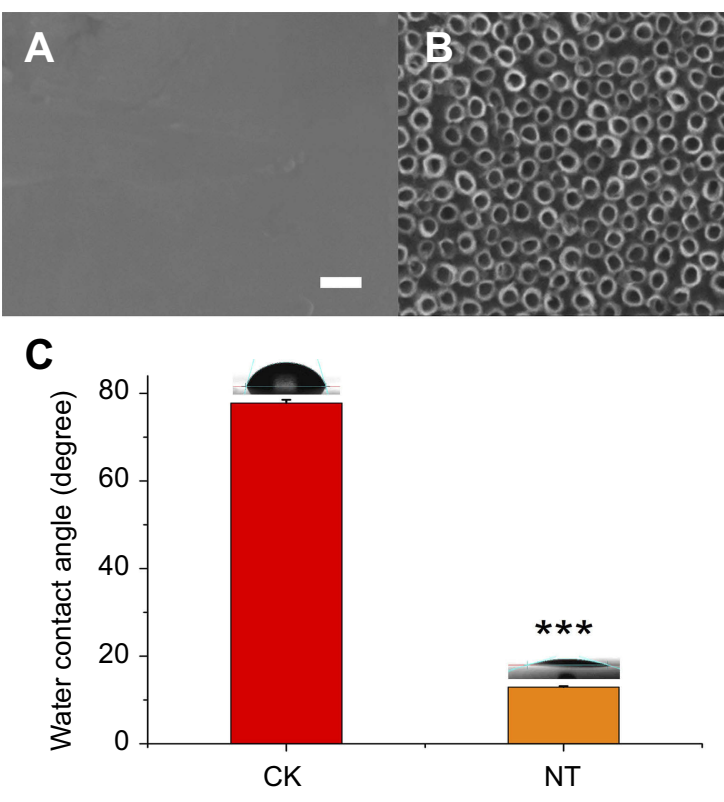

Figure I SEM images of the planar control nitinol (A) and the upright nanotubular coating (B). Scale bars: $100 \mathrm{~nm}$. (C) The decreased water contact angle of the nanotubular coating (NT) compared with that of the planar control nitinol (CK). The data are expressed as the mean \pm standard deviation $(n=4, * * * p<0.00$ I, compared with Student's $t$-test.) 
coatings (NT) by staining the cells with phalloidin and DAPI. We then compared the organization of the actin structures on the nanotubular coatings with those on the planar nitinol surfaces. Confocal microscopy showed that the cells on the flat surfaces were spread out, however, cells on nanotubular coatings had elongated morphologies (Figure 2A and B). The aspect ratio of cells in the NT group was significantly higher than that of cells in the $\mathrm{CK}$ group (Figure 2C). It has been reported that elongation can be a response to mechanical forces induced by topographical cues. ${ }^{23}$ Moreover, a higher migration speed has been observed in elongated cells compared with spread cells. ${ }^{8}$ Based on this evidence, nanotubular topography may facilitate the migration of HCAECs, resulting in rapid re-endothelialization, which is pivotal for wound healing after device implantation.

The phenotypic expression of the endothelial cell marker CD31, the focal adhesion protein vinculin, and the mature endothelial cell marker vWF was confirmed in cells on two different substrate surfaces (Figure S2). HCAECs on the two different substrate surfaces exhibited positivity for CD31, vinculin, and vWF staining. It was assumed that HCAECs cultured on the nitinol-based nanotubular coatings could retain the typical EC phenotype and express adhesion molecules normally.
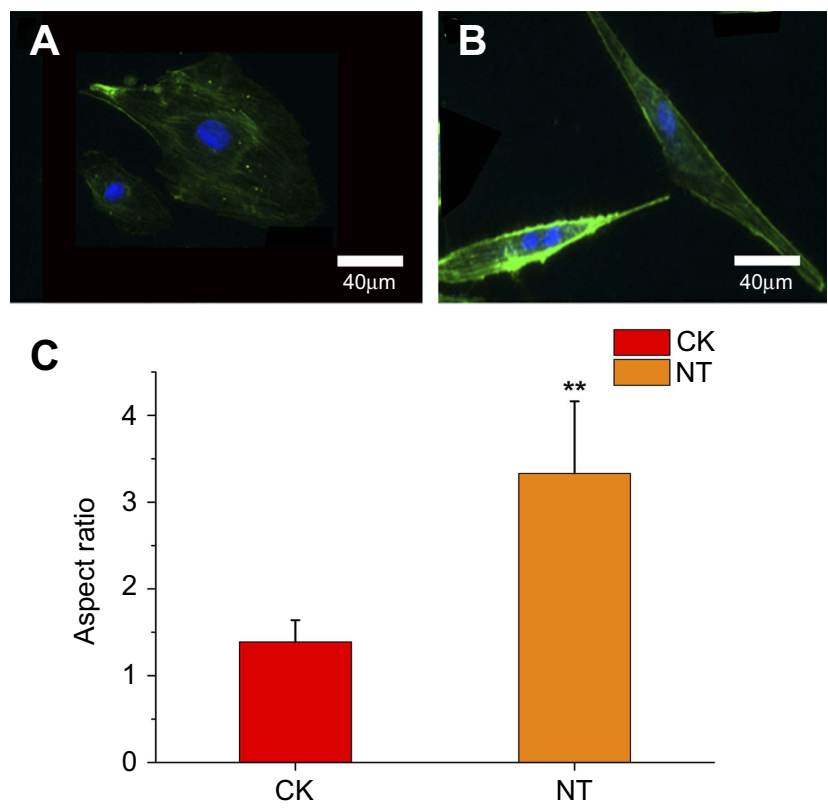

Figure 2 F-actin (green) and nuclear (blue) stains of HCAECs grown for $24 \mathrm{~h}$ on (A) planar surfaces versus (B) nitinol-based nanotubular coatings. (C) Elongation of HCAECs as measured by the aspect ratio. The data are presented as the mean \pm standard deviation $(n=10)$. $* * p<0.01$ significantly different from the CK group, Student's $t$-test (two tailed).

\section{Differentially expressed IncRNAs}

Microarray analysis was performed to investigate the expression levels of lncRNAs in HCAECs on nanotubular coatings compared with those of lncRNAs in cells on control planar nitinol. In total, we identified 1085 significantly differentially expressed lncRNAs in the HCAECs cultured on nanotubular coatings; 687 were upregulated, while 398 were downregulated ( $\geq 2.0$-fold, $p<0.05)$. The 40 most significantly differentially expressed lncRNAs can be found in Table S2 Among these differentially expressed lncRNAs, NONHSAT099739.2 (lnc-TRIML2-8:1) was the most upregulated, with an FC of 10.71, whereas NONHSAT 178380 was the most downregulated, with an FC of 6.90. A scatter plot performed to show the variation in lncRNA expression between the cells cultured on the nanotubular coatings and those cultured on the control planar nitinol (Figure 3A). The clustering analysis was applied to show the relationships among lncRNA expression patterns in the cells cultured on the different topographies (Figure 4A).

\section{Differentially expressed mRNAs}

Microarray analysis identified 227 mRNAs that were significantly differentially expressed in the cells cultured on the nanotubular coatings but not in the cells cultured on control planar nitinol; 136 were upregulated, while 91 were downregulated ( $\geq 2.0$-fold, $p<0.05$ ). The 40 most significantly differentially expressed mRNAs can be found in Table S3. Among the differentially expressed mRNAs, the most significantly upregulated and downregulated mRNAs were CD8B (NM_004931) and PRSS46 (NM_001205271), with FCs of 8.81 and 6.40 , respectively. A scatter plot performed to show the variation in mRNA expression between the cells cultured on the nanotubular coatings and those cultured on control planar nitinol (Figure 3B). The clustering analysis was applied to show the relationships among mRNA expression patterns in the cells cultured on the different topographies (Figure 4B). Protein-protein interaction (PPI) network analysis is used to recognize critical hub members among a cluster of molecules. Accordingly, PPI network analysis was applied to identify critical members among the differentially expressed mRNAs according to the GenOntology, KEGG, Pfam and InterPro databases (Figure S3). 

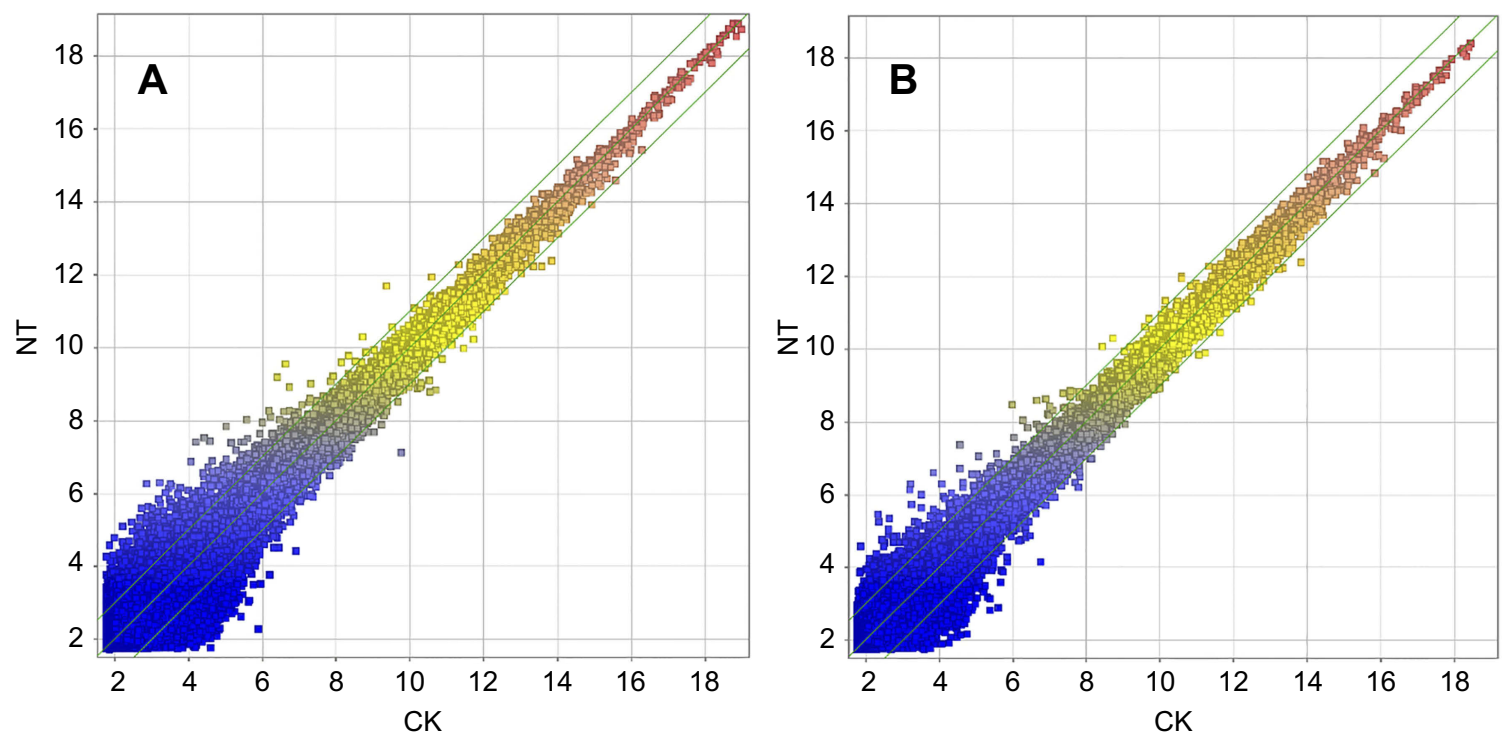

Figure 3 Scatter plot of the variation in IncRNA (A) and mRNA (B) expression between the NT and control samples. The values shown on the $x$-axis and $y$-axis are the normalized signal values of each sample (log 2 scale). The green lines represent FCs (the default fold change value was 2.0). The IncRNAs and mRNAs above the top green line and below the bottom green line had FC values $>2.0$ between the 2 compared samples.

\section{Validation of the microarray data using qRT-PCR}

qRT-PCR was performed to confirm the microarray analysis findings. Four differentially expressed lncRNAs (lnc-MYO1B-1:1, lnc-TRIML2-8:1, NONHSAT178380, and NONHSAT190776) were randomly selected for validation. The expression levels of these lncRNAs are shown in Figure 5. In accordance with the microarray analysis, the results of qRT-PCR demonstrated that lncMYO1B-1:1 and lnc-TRIML2-8:1 were upregulated in the NT group compared with the CK group, while NONHSAT178380 and NONHSAT190776 were downregulated.

\section{Microarray-based GO analysis and pathway analysis}

Functional analyses for differentially expressed mRNAs were performed by GO and pathway analyses. In this study, we found that some of the most important GO terms were related to cell migration. For example, the most enriched GO terms in the upregulated transcripts were "exocyst localization" (ontology aspect: biological process, Figure S4) and "integral component of plasma membrane" (ontology aspect: cellular component, Figure S5). Among the downregulated transcripts, some of the most enriched GO terms were "cell-cell signaling" (ontology aspect: biological process, Figure S6), "extracellular region" (ontology aspect: cellular component, Figure S7) and "somatostatin receptor activity" (ontology aspect: molecular function, Figure S8). We also obtained the 10 GO terms that were most associated with the gene coding of upregulated RNAs and downregulated RNAs in the molecular function, biological process, and cellular component domains (Figure S9, S10, and S11). KEGG pathway analysis showed that a total of 111 pathways were related to the upregulated transcripts. However, we found that 123 pathways were related to the downregulated transcripts, and the pathway with the most enrichment was "ECM-receptor interaction" (Figure S12). The mRNA expression levels of three genes in "ECM-receptor interaction" pathway are shown in Figure 6. The results of qRT-PCR demonstrated that COL4A5 and COL9A3 expression did not differ significantly in the NT group compared with the levels in the $\mathrm{CK}$ group, while COL9A1 was downregulated in the NT group. Our results also showed that the top 20 pathways were associated with the genes encoding differentially expressed RNAs (Figure S13). These results indicated that "ECM-receptor interaction" and "cell adhesion molecules" are significant pathways in HCAECs induced by nanotubular topography.

\section{Construction of the coexpression network}

A gene coexpression network was constructed with the differentially expressed lncRNAs and mRNAs with absolute values of the Pearson correlation coefficient 

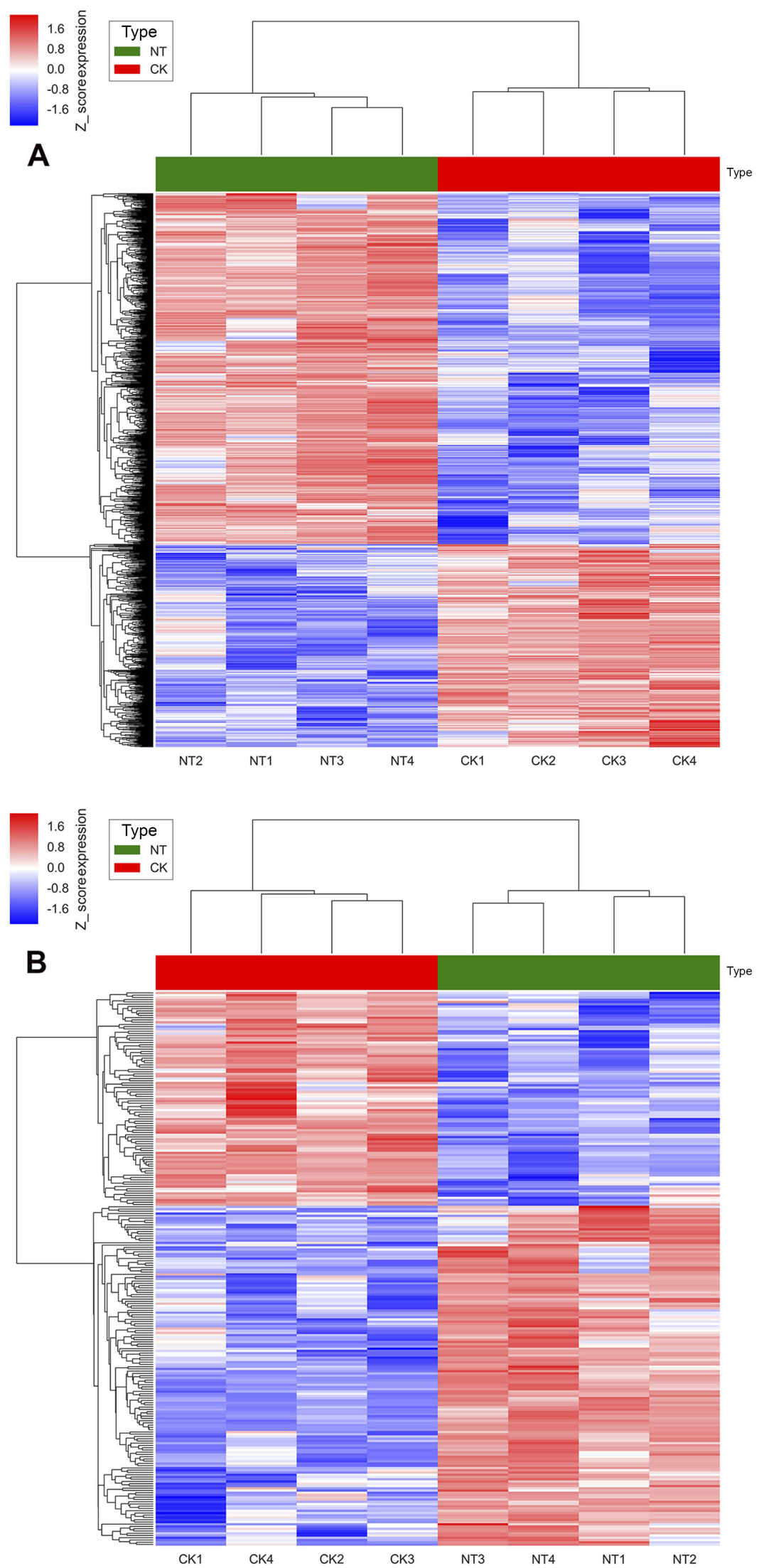

Figure 4 (A) Hierarchical clustering analysis of 1085 differentially expressed IncRNA probe sets. (B) Hierarchical clustering analysis of 227 differentially expressed mRNA probe sets. The samples are in the columns, and the probe sets are in the rows. 


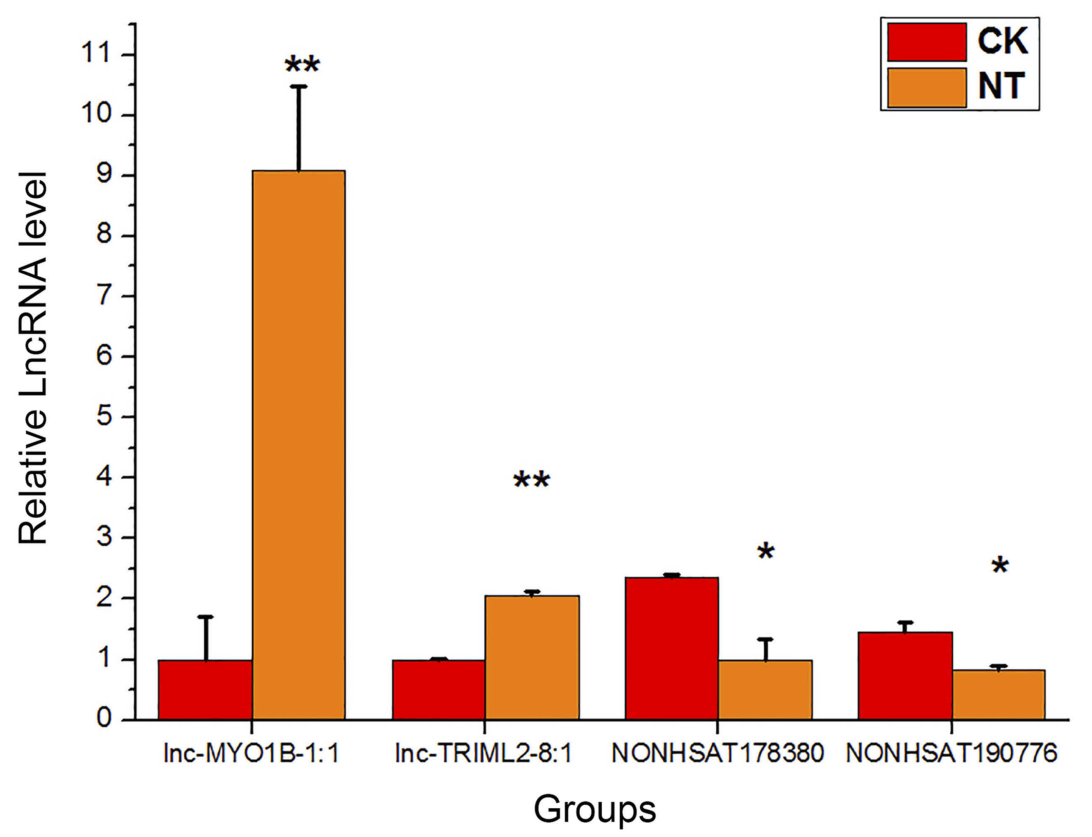

Figure 5 qRT-PCR validation results of four randomly selected IncRNAs. The target IncRNA expression levels were normalized to the internal control GAPDH. The data are presented as the mean \pm standard deviation $(n=3) \cdot{ }^{*} p<0.05,{ }^{* *} p<0.0$ I significantly different from the CK group, Student's $t$-test (two tailed).

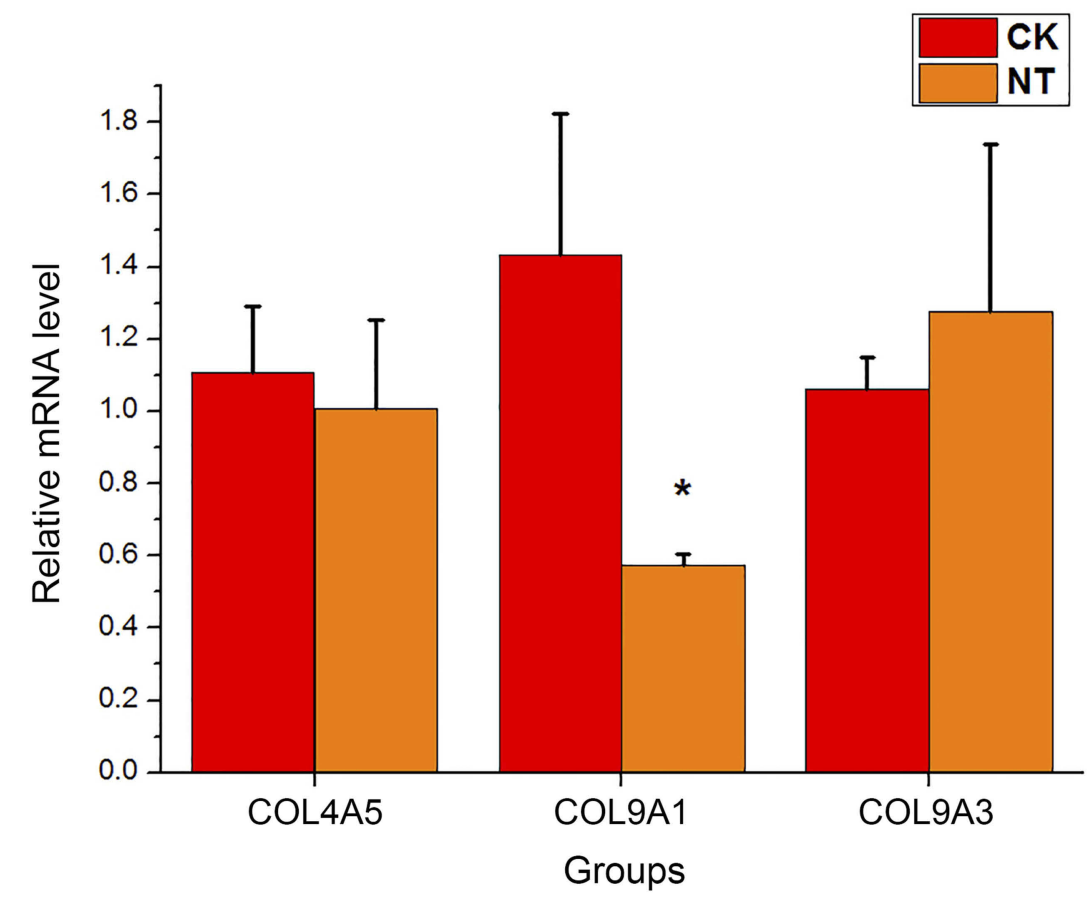

Figure 6 The mRNA expression levels of three genes involved in "ECM-receptor interaction" pathway. The target mRNA expression levels were normalized to the internal control GAPDH. The data are presented as the mean \pm standard deviation $(n=3){ }^{*} p<0.05$ significantly different from the CK group, Student's $t$-test (two tailed).

$>0.9$ and $p<0.05$ (Figure S14), which consisted of 53 nodes and 92 connections between 10 lncRNAs and 43 mRNAs. In this network, 36 pairs presented as positive, and 56 pairs presented as negative. $p$-values were calculated for the enrichment between differentially expressed lncRNAs and TFs using the hypergeometric distribution method. As shown in Figure 7, the data illustrated that most of these potential transregulatory 


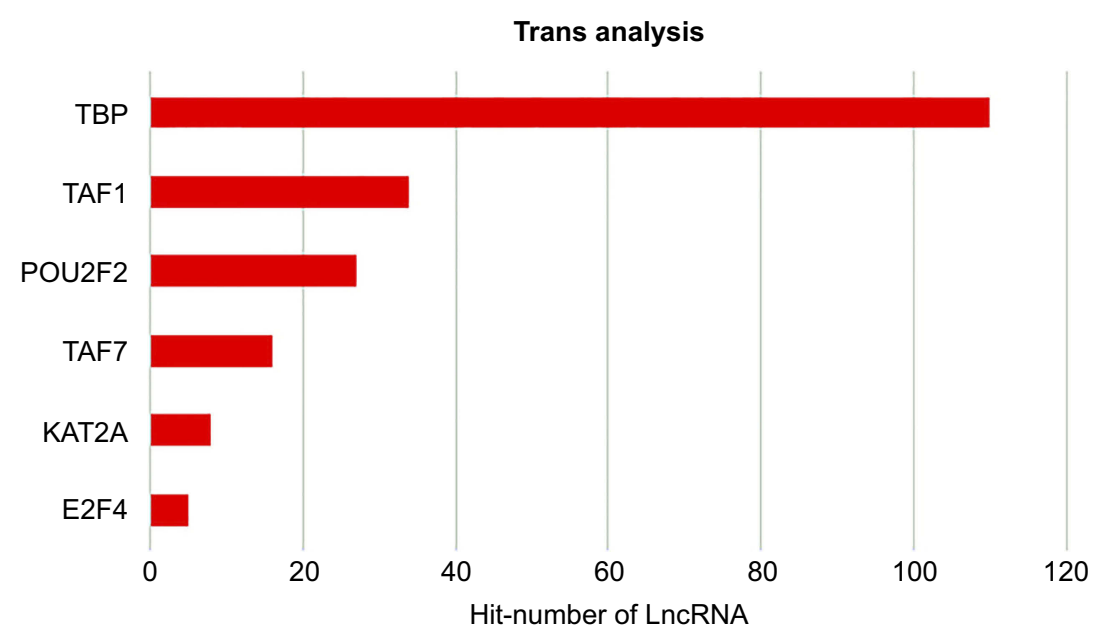

Figure 7 Top-ranking TFs associated with the differently expressed IncRNAs according to $p$-value.

lncRNAs participate in pathways regulated by 5 TFs: TBP, TAF1, POU2F2, TAF7 and KAT2A. Our analysis also revealed the TFs most associated with the differentially expressed lncRNAs (Figure 8). On the basis of lncRNA-TF-mRNA analysis (Figure 9), TBP (TATAbinding protein) was identified as a transcription regulator with a critical role in the molecular pathways. TBP is a protein that binds to a consensus TATA-box sequence element and is found in transcription factor complexes involved in RNA polymerase I-, II- and IIImediated transcription. Small increases in cellular TBP levels have been found to induce changes in cellular growth properties toward a transformed phenotype, depending on the cell type. ${ }^{24}$ miRNAs, another class of noncoding RNA molecules, play versatile roles in many biological processes. Figure 10 presents a miRNA-lncRNA-

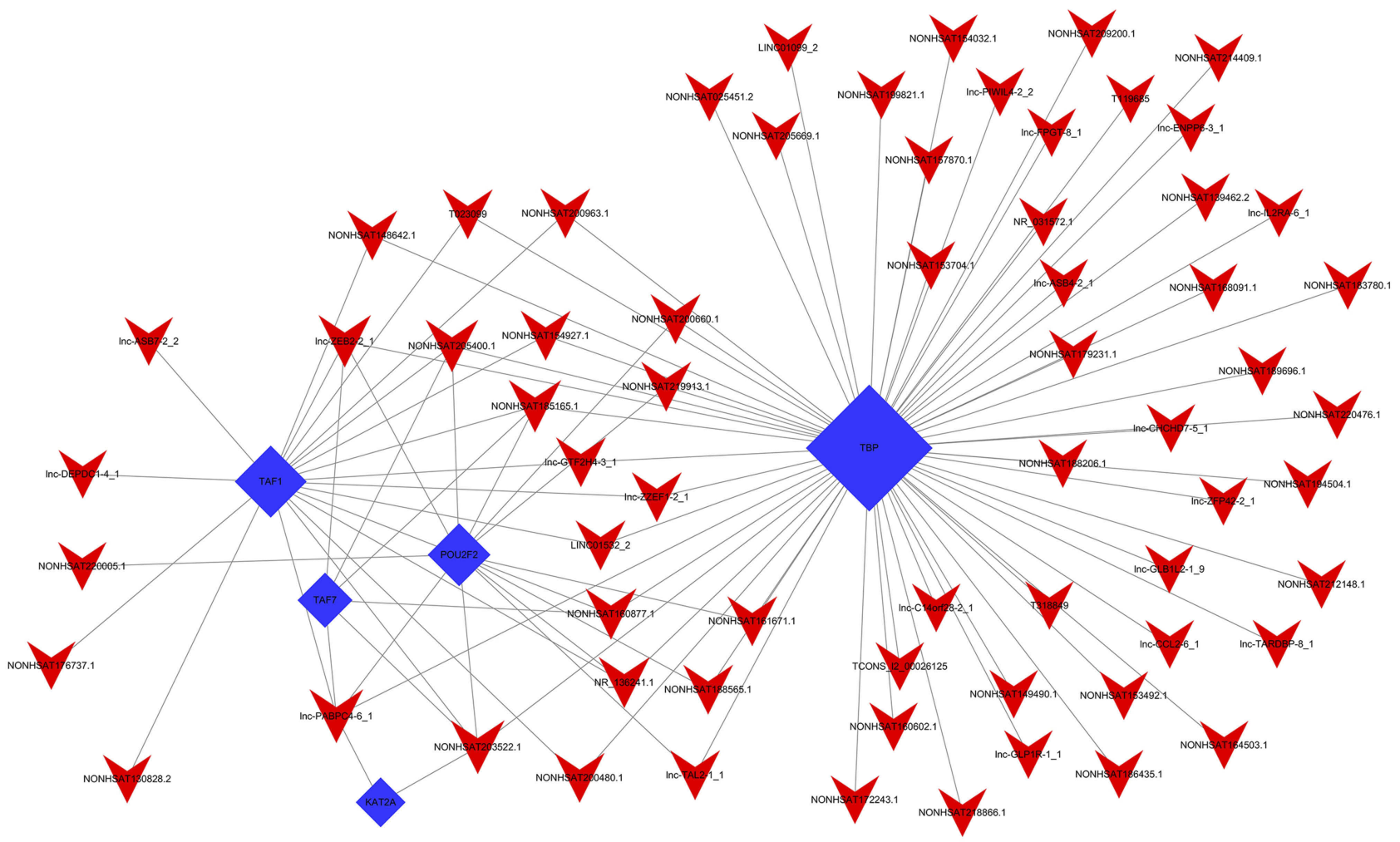

Figure 8 IncRNA-TF network visualized by hypergeometric distribution analysis. The top 100 IncRNA-TF relationships sorted by $p$-value were selected to construct a dyadic relationship network. The blue and red nodes represent the TFs and IncRNAs, respectively. 


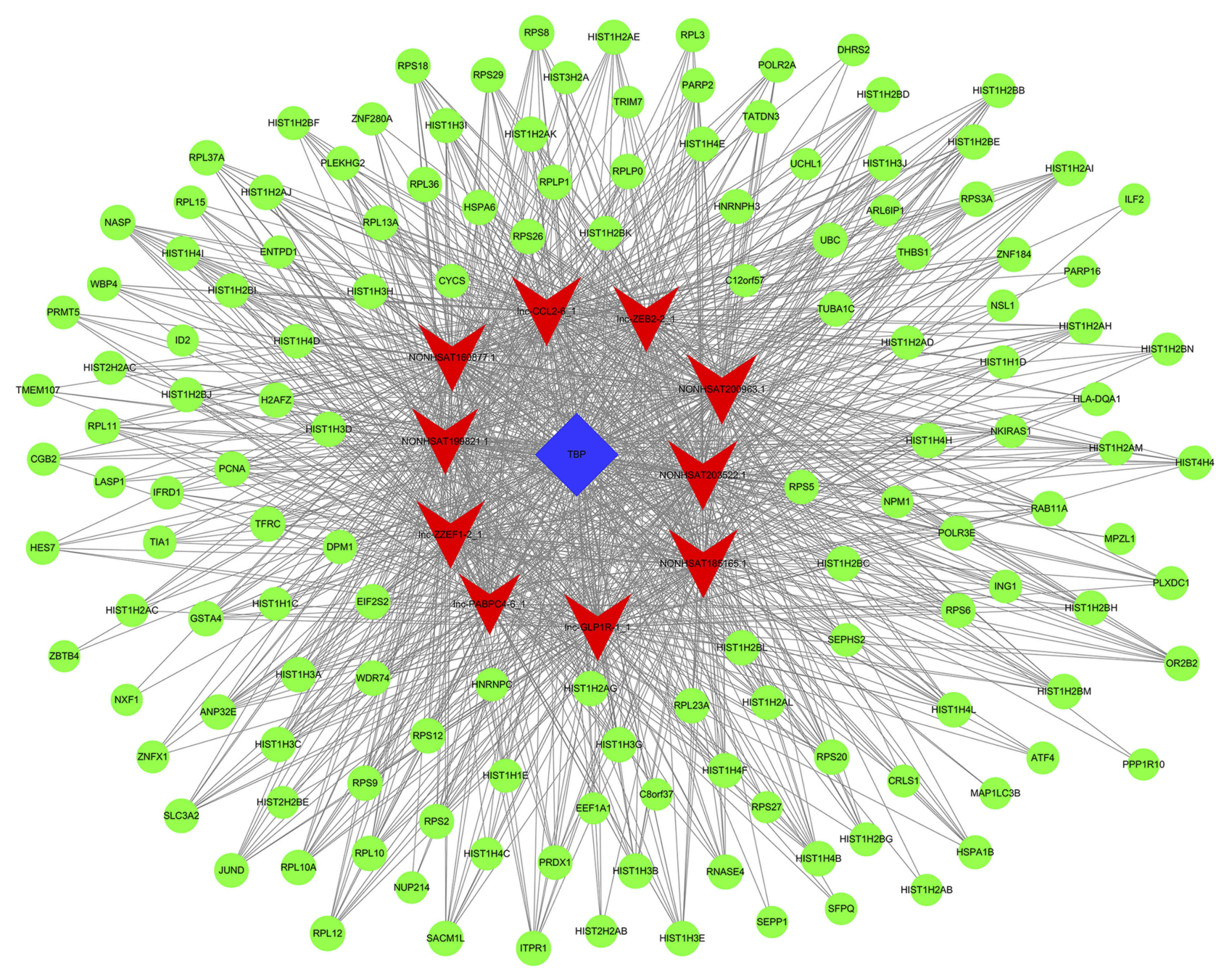

Figure 9 IncRNA-TF-mRNA core network consisting of the 996 pairs of IncRNAs, TFs, and mRNAs with the most relevance. The red nodes represent the IncRNAs, the blue rectangles represent the TFs, and the green circles represent the mRNAs.

mRNA coexpression network according to the differentially expressed miRNAs, lncRNAs, and mRNAs. The results showed that the differentially expressed lncRNAs and mRNAs interacted with 20 miRNAs in HCAECs in response to nitinol-based nanotubular coatings.

\section{Discussion}

In the present study, we investigated, for the first time, the altered expression pattern of lncRNAs in HCAECs in response to nitinol-based nanotubular coatings using microarray analysis. Also, potential roles and molecular networks of differentially expressed mRNAs and lncRNAs were predicted. In total, 1085 lncRNAs and 227 mRNAs were differentially expressed owing to the nitinol-based nanotubular coatings.

To gain insight into the potential functions of the differentially expressed mRNAs, GO and pathway analyses were performed. The most significantly altered categories identified by $\mathrm{GO}$ analysis were those involving the plasma membrane and exocyst-related processes. It has been shown that the exocyst interacts with Rho GTPases resulting in cell polarity and the activity of the cytoskeleton. ${ }^{25}$ In addition to regulation by the cytoskeleton, integrin activation is also regulated by small GTPases. ${ }^{26}$ Integrins are the major cell-ECM adhesion receptors. They are also mechanotransducers that can modulate cytoskeletal remodeling through the regulation of Rho family small GTPases. ${ }^{26}$ It is suggested that the nanotubular topography influences the interaction between integrins and Rho GTPases in HCAECs through plasma membrane and exocyst-related processes, leading to the change of cytoskeleton organization. In addition, GO analysis identified the most important upregulated molecular functions as G-protein coupled receptor activity, calmodulin-dependent protein kinase activity and calcium ion binding, all of which have 


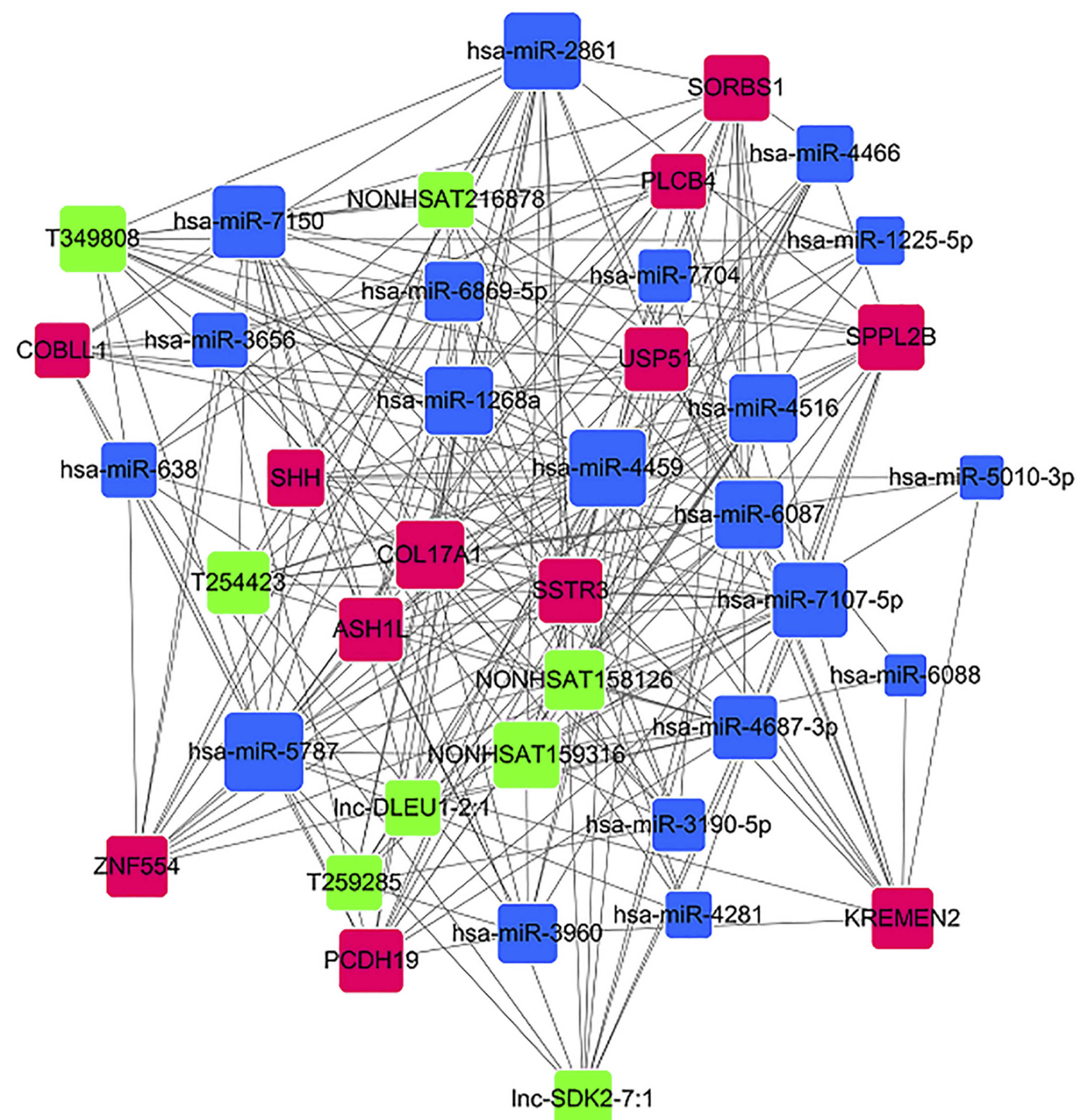

Figure 10 miRNA-IncRNA-mRNA coexpression network. The red rectangles represent the mRNAs, the blue rectangles represent the miRNAs, and the green rectangles represent the IncRNAs.

previously been implicated in mechanotransduction. ${ }^{27}$ It has been reported that $\mathrm{Ca}^{2+}$ signaling can activate G-proteins via a mechanical signal. ${ }^{28}$ G-proteins can also regulate mechanotransduction and activation of specific $\mathrm{G}$ proteins may modulate the activity of specific Rho GTPases, ${ }^{26}$ which can interact with exocyst participating in exocyst-related processes mentioned above. Previous studies have suggested that increased roughness on stainless steel surfaces may improve the expression of cell adhesion molecules in ECs such as E-selectin and VCAM- $1 ;{ }^{29}$ the most highly correlated pathways that we identified were those related to ECM-receptor interaction and cell adhesion molecules. Therefore, we speculate that the nanotubular topography can affect the cell adhesion molecules located on the surface of the cell membrane, and regulate the behavior and function of endothelial cells by downstream G-protein coupled receptors and $\mathrm{Ca}^{2+}$ signaling. However, all of these speculations need to be studied in future work.

Among the top-ranking transfactors associated with the differentially expressed lncRNAs, TBP and TAF1 are the most important. It has been reported that TBP levels regulate the expression of VEGFA, which is a ratelimiting growth factor for angiogenesis. Furthermore, improvement of cellular TBP levels contribute to VEGFA expression and secretion involved in enhancing cell migration. ${ }^{24}$ Whether it is possible for nanotubular topography to regulate the behavior of endothelial cells via the expression of TBP and VEGFA is also worthy of 
further exploration. We also identified another significant transfactor, E2F4, that was associated with the differently expressed lncRNAs. E2F4 belongs to the E2F family, which is a series of transcription factors involved in cell proliferation in response to extra- and intra-cellular signals. $^{30}$ It has been shown that E2F3 promotes the proliferation of vascular smooth muscle cells, resulting in increased intimal hyperplasia, whereas E2F4 inhibits this pathological response. ${ }^{31}$ Therefore, further investigation of the potential roles of E2F4-associated differently expressed IncRNAs in intimal hyperplasia inhibition is warranted. Overall, we speculate that the transcription factors TBP and TAF1 in the nucleus regulate the expression of certain genes contributing to changes in cell function. The functions of TBP and TAF1 involved in EC responses to substrate topography need to be proved by further experiments.

However, one limitation of this study is that the effect of shear stress on blood flow was not explored. Blood flow shear stress is also an important factor influencing endothelial cells after stent implantation.Shear stress has an intense effect on the function and behavior of vascular endothelial cells, so future research should explore the changes in lncRNA expression when the cells are subjected to shear stress and substrate topography together. In addition, it has been proved that smooth muscle cells are also an important cell type after stenting, so we will focus on such cells in further research. By comparing the gene expression between endothelial cells and smooth muscle cells, surfaces of vascular stents that are more favorable to endothelial cell proliferation and function can be designed, and the function of vascular stents can be further promoted.

\section{Conclusion}

In this work, a microarray analysis was performed to confirm the 1085 lncRNAs differentially expressed in HCAECs in response to nitinol-based nanotubular coatings. Potential roles and molecular networks of these differentially expressed mRNAs and lncRNAs were also predicted. Extracellular matrix receptor interactions and cell adhesion molecules play critical roles in the sensing of nitinol-based nanotubular coatings by HCAECs. TBP and TAF1 are important molecules in EC responses to substrate topography, but their functions involved in EC responses to nanotubular topography need to be proved by further experiments. Identifying the lncRNAs and networks involved in the responses to nanotopographical cues may be crucial in understanding how cells respond to nanotopography. Furthermore, revealing how cells and topography interact can improve the design of future medical devices.

\section{Acknowledgments}

This work was supported by grants from the National Natural Science Foundation of China (No. 81600148, 51621003, U1607110) and the Beijing Municipal Commission of Education Foundation (KZ201610005002).

\section{Disclosure}

The authors declare that they have no competing interests in this work.

\section{References}

1. Forouzanfar MH, Moran AE, Flaxman AD, et al. Assessing the global burden of ischemic heart disease, part 2: analytic methods and estimates of the global epidemiology of ischemic heart disease in 2010 . Glob Heart. 2012;7(4):331-342. doi:10.1016/j.gheart.2012.10.003

2. Di NM, Middeldorp S, Büller HR. Direct thrombin inhibitors. New England Journal of Medicine. 2005;353(10):1028. doi:10.1056/ NEJMra044440

3. Avci-Adali M, Ziemer G, Wendel HP. Induction of EPC homing on biofunctionalized vascular grafts for rapid in vivo selfendothelialization-a review of current strategies. Biotechnol Adv. 2010;28(1):119-129. doi:10.1016/j.biotechadv.2009.10.005

4. Castellanos MI, Guillem-Marti J, Mas-Moruno C, et al. Cell adhesive peptides functionalized on $\mathrm{CoCr}$ alloy stimulate endothelialization and prevent thrombogenesis and restenosis. J Biomed Mater Res A. 2017;105(4):973. doi:10.1002/jbm.a.35988

5. Bettinger CJ, Langer R, Borenstein JT. Engineering substrate topography at the micro- and nanoscale to control cell function. Angewandte Chemie. 2010;48(30):5406-5415. doi:10.1002/ anie. 200805179

6. Tan CH, Muhamad N, Abdullah MMAB Surface topographical modification of coronary stent: a review. IOP Conference Series Materials Science and Engineering. 2017;209(1):012-031. doi:10.1088/1757899X/209/1/012031

7. Peng L, Eltgroth ML, Latempa TJ, Grimes CA, Desai TA. The effect of $\mathrm{TiO}_{2}$ nanotubes on endothelial function and smooth muscle proliferation. Biomaterials. 2009;30(7):1268-1272. doi:10.1016/j. biomaterials.2008.11.012

8. Brammer KS, Oh S, Gallagher JO, Jin S. Enhanced cellular mobility guided by $\mathrm{TiO}_{2}$ nanotube surfaces. Nano Lett. 2008;8(3):786-793. doi:10.1021/n1072572o

9. Zhong S, Luo R, Wang X, et al. Effects of polydopamine functionalized titanium dioxide nanotubes on endothelial cell and smooth muscle cell. Colloids Surf B Biointerfaces. 2014;116(5):553-560. doi:10.1016/j.colsurfb.2014.01.030

10. Lee PP, Cerchiari A, Desai TA. Nitinol-based nanotubular coatings for the modulation of human vascular cell function. Nano Lett. 2014;14(9):5021-5028. doi:10.1021/n1501523v

11. Archer K, Broskova Z, Bayoumi AS, et al. Long non-coding RNAs as master regulators in cardiovascular diseases. Int J Mol Sci. 2015;16(10):23651-23667. doi:10.3390/ijms 161023651

12. Gomes CPC, Spencer H, Ford KL, et al. The function and therapeutic potential of long non-coding RNAs in cardiovascular development and disease. Mol Ther Nucleic Acids. 2017;8(C):494-507. doi:10.1016/j.omtn.2017.07.014 
13. Boon RA, Holdt L, Dimmeler S. Long noncoding RNAs: from clinical genetics to therapeutic targets? J Am Coll Cardiol. 2016;67 (10):1214-1226. doi:10.1016/j.jacc.2015.12.051

14. Zhou T, Ding JW, Wang XA, Zheng X-X. Long noncoding RNAs and atherosclerosis. Atherosclerosis. 2016;248:51-61. doi:10.1016/j. atherosclerosis.2016.02.025

15. Wang N, Li H, Lü W, et al. Effects of TiO nanotubes with different diameters on gene expression and osseointegration of implants in minipigs. Biomaterials. 2011;32(29):6900-6911. doi:10.1016/j. biomaterials.2011.06.023

16. Lv L, Liu Y, Zhang P, et al. The nanoscale geometry of $\mathrm{TiO}_{2}$ nanotubes influences the osteogenic differentiation of human adipose-derived stem cells by modulating H3K4 trimethylation. Biomaterials. 2015;39 (Complete):193-205. doi:10.1016/j.biomaterials.2014.11.002

17. Wenwen L, Penglei S, Su C, et al. Synthesis of $\mathrm{TiO}_{2}$ nanotubes with $\mathrm{ZnO}$ nanoparticles to achieve antibacterial properties and stem cell compatibility. Nanoscale. 2014;6(15):9050. doi:10.1039/C4NR01531B

18. Guttman M, Amit I, Garber M, et al. Chromatin signature reveals over a thousand highly conserved large non-coding Rnas in mammals. Nature. 2009;458:223-227. doi:10.1038/nature07672

19. Mitchell G, Julie D, Carey BW, et al. IncRNAs act in the circuitry controlling pluripotency and differentiation. Nature. 2011;477 (7364):295. doi:10.1038/nature10398

20. Alexander S, Johannes KS, Tobias M, et al. Next-generation RNA sequencing reveals differential expression of $\mathrm{MYCN}$ target genes and suggests the mTOR pathway as a promising therapy target in MYCN-amplified neuroblastoma. Int J Cancer. 2012;132(3):E106E115. doi:10.1002/ijc. 27787

21. Wilson CJ, Clegg RELeavesley DI, Pearcy MJ. Mediation of biomaterial-cell interactions by adsorbed proteins: a review. Tissue Eng. 2005;11(2):1-18. doi:10.1089/ten.2005.11.1
22. Hersel U, Dahmen C, Kessler H. RGD modified polymers: biomaterials for stimulated cell adhesion and beyond. Biomaterials. 2003;24 (24):4385-4415

23. Sato M, Ohashi T. Biorheological views of endothelial cell responses to mechanical stimuli. Biorheology. 2005;42(6):421.

24. Sas J, Lin JJ, Walkey CJ, Leathers MP, Coarfa C, Johnson DL. Elevated TATA-binding protein expression drives vascular endothelial growth factor expression in colon cancer. Oncotarget. 2017;8 (30):48832-48845. doi:10.18632/oncotarget.16384

25. Ory S, Gasman S. Rho GTPases and exocytosis: what are the molecular links? Semin Cell Dev Biol. 2011;22(1):27-32. doi:10.1016/j. semcdb.2010.12.002

26. Song L, Huang NF, Steven H. Mechanotransduction in endothelial cell migration. J Cell Biochem. 2010;96(6):1110-1126.

27. Jaalouk DE, Lammerding J. Mechanotransduction gone awry. Nat Rev Mol Cell Biol. 2009;10(1):63. doi:10.1038/nrm2597

28. Plank MJ, Wall DJN, David T. Atherosclerosis and calcium signalling in endothelial cells. Prog Biophys Mol Biol. 2006;91 (3):287-313. doi:10.1016/j.pbiomolbio.2005.07.005

29. Mclucas E, Moran MT, Rochev Y, Carroll WM, Smith TJ. An investigation into the effect of surface roughness of stainless steel on human umbilical vein endothelial cell gene expression. Endothelium J Endoth Cell Res. 2006;13(1):35. doi:10.1080/ 10623320600660185

30. Hsu J, Sage J. Novel functions for the transcription factor E2F4 in development and disease. Cell Cycle. 2016;15(23):3183-3190. doi:10.1080/15384101.2016.1234551

31. Giangrande PH, Zhang J, Tanner A, et al. Distinct roles of E2F proteins in vascular smooth muscle cell proliferation and intimal hyperplasia. Proc Natl Acad Sci. 2007;104(32):12988-12993. doi:10.1073/pnas.0704754104
International Journal of Nanomedicine

\section{Publish your work in this journal}

The International Journal of Nanomedicine is an international, peerreviewed journal focusing on the application of nanotechnology in diagnostics, therapeutics, and drug delivery systems throughout the biomedical field. This journal is indexed on PubMed Central, MedLine, CAS, SciSearch $₫$, Current Contents $₫ /$ Clinical Medicine,
Journal Citation Reports/Science Edition, EMBase, Scopus and the Elsevier Bibliographic databases. The manuscript management system is completely online and includes a very quick and fair peer-review system, which is all easy to use. Visit http:/www.dovepress.com/ testimonials.php to read real quotes from published authors. 